\title{
Molecular Modeling Analysis of Chitosan-Dopamine Blend with Iron Oxide Nanoparticles for Tissue Engineering Applications
}

\author{
Nayera El-Sayed ${ }^{1(\mathbb{D})}$, Mohammed El-Bakary ${ }^{1(\mathbb{D})}$, Medhat Ibrahim ${ }^{2, * \mathbb{D}}$, Mohamed ELgamal ${ }^{3(\mathbb{D})}$ \\ 1 Physics Department, Faculty of Science, Mansoura University, Mansoura 35516, Egypt \\ 2 Molecular Spectroscopy and Modeling Unit, Spectroscopy Department, National Research Centre, 33 El-Bohouth St., \\ 12622 Dokki, Giza, Egypt \\ 3 Congenital and Pediatric Cardiac Surgery, Faculty of Medicine, Mansoura University, Mansoura 35516, Egypt \\ * Correspondence: medahmed6@yahoo.com;
}

Received: 11.12.2020; Revised: 20.01.2021; Accepted: 23.01.2021; Published: 30.01.2021

\begin{abstract}
This study aims to mimic mussel adhesive protein (MAPs) by mussel-inspired metalcoordination chemistry at the polymer-particle interface using iron oxide nanoparticles $\left(\mathrm{Fe}_{3} \mathrm{O}_{4} \mathrm{NPs}\right)$ and catechol-polymer as the building blocks. Catechol group of dopamine conjugates with chitosan backbone and provides additional adhesion strength with tissue surfaces. Molecular modeling, including two different methods, Quantitative structure-activity relationship (QSAR) and molecular electrostatic potential (MESP), was used to study the suggested tissue adhesive's physical and structural properties. Four positions of $\mathrm{Fe}_{3} \mathrm{O}_{4} \mathrm{NPs}$ to connect with chitosan-dopamine blend were proposed. The third site was preferred by following the bandgap energy $(\Delta \mathrm{E})$ results and the total dipole moment (TDM).
\end{abstract}

Keywords: chitosan; dopamine; iron oxide nanoparticles; QSAR and MESP.

(c) 2021 by the authors. This article is an open-access article distributed under the terms and conditions of the Creative Commons Attribution (CC BY) license (https://creativecommons.org/licenses/by/4.0/).

\section{Introduction}

In recent years there has been an increased interest in replacing sutures and staples used in surgical procedures with adhesive bonds and synthetic sealants. Also, conventional wound closure procedures have several potential limitations. For example, fluid leakage during the suturing process and the cuts or holes created by these sutures lead to various infections $[1,2]$. So, researchers have attempted to develop the mechanical and structural properties of different types of sutures $[3,4]$. Other researchers have also made many research papers to improve various sealants and adhesives to bond tissue in a wide range of surgical procedures. Tissue adhesives have been used to close the wounds and prevent leaks as an alternative and/or supporting the sutures [5]. An adhesive spreads over the entire contact area, eliminating stress localization facilitating load transfer between the fractured surfaces [6]. Although the designed tissue adhesives to facilitate wound healing are gaining growing popularity in diverse clinical applications, they present significant inherent limitations such as rejection, infections, toxicity, and excessive swelling [7]. Furthermore, the performance of adhesives is dramatically reduced underwater or in moisturized conditions. Nature has been a powerful source of inspiration in the design and engineering of novel biomaterials for many years. The successful strategy to design novel synthetic materials is to mimic the production processes found in nature. Marine 
mussels are known to adhere to various substrates under wet conditions. An unusual amino acid, 3,4-dihydroxy-L-phenylalanine (DOPA), is a modified amino acid found in marine organisms [8-11]. This study's idea is that a biomimetic tissue adhesive based on mussel adhesive protein certainly by using 3,4-dihydroxy-L-phenylalanine (DOPA) is responsible for both the speedy curing of the adhesive and interfacial binding [12]. Chitosan has excellent biological properties presenting a promising prospect in antibacterial and antioxidant biomaterials with little cytotoxicity [13]. Chitosan-based agents have shown great promise in controlling major hemorrhaging in a pre-hospital setting. However, the tissue adhesion ability of chitosan hydrogels is limited because chitosan hydrogel weakly interacts with tissue. Therefore, the catechol group of dopamine conjugates with the chitosan backbone and provides additional adhesion strength with tissue surfaces [14]. Various compounds such as chitosan and dopamine have been coated with various types of nanoparticles (NPs) to promote tissue cross-linking and ensure a non-reversible chemical bond under wet physiological conditions [15]. In addition to improving the mechanical properties and adhesive strength. Inorganic nanoparticles are known as tissue adhesives owing to the so-called "Nano-bridging effect" between numerous protein chains in the wound. There is a novel approach to assemble nanocomposite hydrogels via mussel-inspired metal-coordination chemistry at the polymer-particle interface using iron oxide nanoparticles $\left(\mathrm{Fe}_{3} \mathrm{O}_{4} \mathrm{NPs}\right)$ and catechol-polymer as the building blocks. As a result of the catechol-Fe (III) interfacial bond dynamics and the unique network structure, the assembled polymer-nanoparticle composite demonstrates strikingly different relaxation mechanics compared to conventional covalent cross-linked gels $[5,10]$. Molecular modeling and other computational simulation methods have proved their capabilities in studying the properties and interactions between different chemical structures and provide reliable data concerning all biological, chemical, and physical features [16-21]. QSAR methodology is a computational tool that can quantify the relationship between a certain biological structure's physicochemical properties and its biological activities [22-24]. Furthermore, there is another valuable concept in molecular modeling calculations that offers entirely accurate information on several chemical entities' effective sites. This concept is known as Molecular Electrostatic Potential (MESP). It is also critical in estimating the chemical addition nature through which a chemical structure is most possible to go through, either the electrophilic or nucleophilic chemical addition. Molecular modeling is generally widely applied to study structural, thermal, and vibrational features of many molecules [2529]. Physical properties such as MESP, total dipole moment, HOMO/LUMO bandgap energy reflect the studied structures' reactivity [30-33]. Accordingly, these parameters are calculated as a descriptor for the reactivity of the studied structures. In this study, a prediction of the structural model of chitosan with dopamine was investigated to understand a way of the functionalization process. Furthermore, molecular modeling at B3LYP/6-31G (d,p) method was utilized to study the effect of $\mathrm{Fe}_{3} \mathrm{O}_{4} \mathrm{NPs}$ on the structural properties of the chitosandopamine blend.

\section{Materials and Methods}

\subsection{Computational details.}

All the studied models were calculated with GAUSSIAN 09, which was implemented at Spectroscopy Department, National Research Center (NRC) [34]. Calculations were conducted with density functional theory DFT using Becke-three-parameters-Lee-Yang-Parr 
hybrid functional (B3LYP) [35-37], utilizing the basis set 6-31G(d,p). First, the structure optimization was done to locate the energy minimum. Then, the studied physical parameters were also calculated at the B3LYP/6-31G $(\mathrm{d}, \mathrm{p})$ level of theory. The studied models' QSAR parameters were evaluated with SCIGRESS 0.3, which was implemented at Spectroscopy Department, National Research Center (NRC), using the PM6 semiempirical method.

\section{Results and Discussion}

\subsection{Building model molecules.}

The suggested models consist of three subunits for chitosan with one dopamine unit as a blend were studied. For this blend, iron oxide nanoparticles $\left(\mathrm{Fe}_{3} \mathrm{O}_{4} \mathrm{NPs}\right)$ were added at four different positions. Model molecules of (a) Chitosan, (b) Dopamine, and (c) blend of chitosan and dopamine are demonstrated in figure 1 . The chitosan-dopamine $\mathrm{OOH}$ interaction is through the hydroxyl group $(\mathrm{OH})$ of the first unit of chitosan oligomer and $(\mathrm{O})$ of dopamine. Fourposition probabilities have suggested the interaction of $\mathrm{Fe}_{3} \mathrm{O}_{4} \mathrm{NPs}$ with a chitosan-dopamine blend.

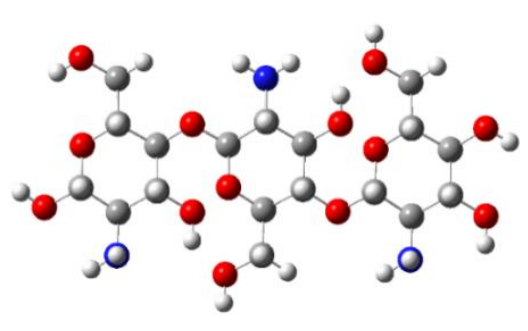

a

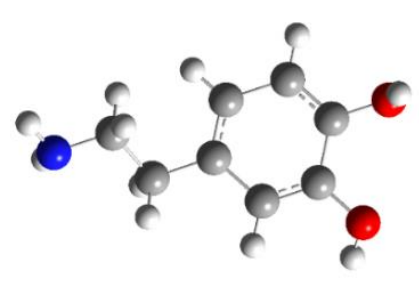

$\mathrm{b}$

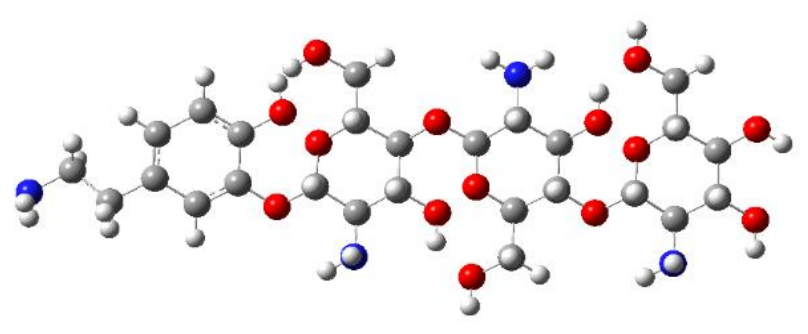

C

Figure 1. Molecular model molecules of chitosan (a), dopamine (b), and a blend of chitosan and dopamine(c) calculated at B3LYP/6-31G (d,p). [C in grey, $\mathrm{H}$ in white grey, $\mathrm{O}$ in red, and $\mathrm{N}$ in blue].

Figure 2 (a-d) demonstrates the four suggested positions of $\mathrm{Fe}_{3} \mathrm{O}_{4}$ NPs interaction, (a) chitosan-dopamine-iron interaction prospect through the hydroxyl group $(\mathrm{OH})$ of the first unit of chitosan oligomer and $(\mathrm{OH})$ of dopamine, (b) chitosan-dopamine-iron interaction prospect through the hydroxyl group $(\mathrm{OH})$ of the first and second unit of chitosan oligomer, (c) chitosandopamine-iron interaction prospect through the hydroxyl group $(\mathrm{OH})$ of the second and third unit of chitosan oligomer, (d) chitosan-dopamine-iron interaction prospect through the hydroxyl group $(\mathrm{OH})$ of the third unit of chitosan. 


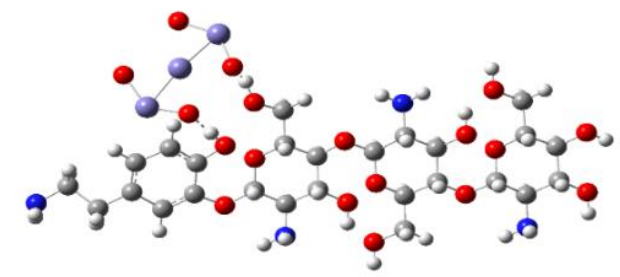

a-

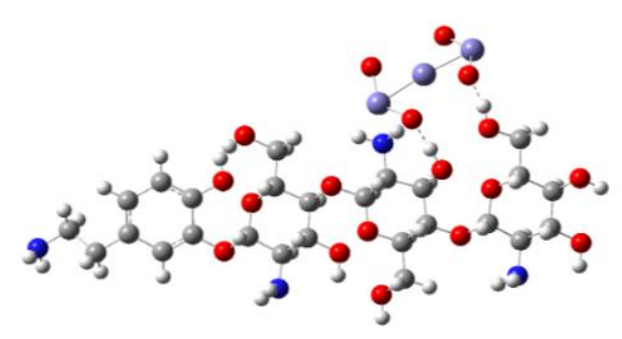

c-

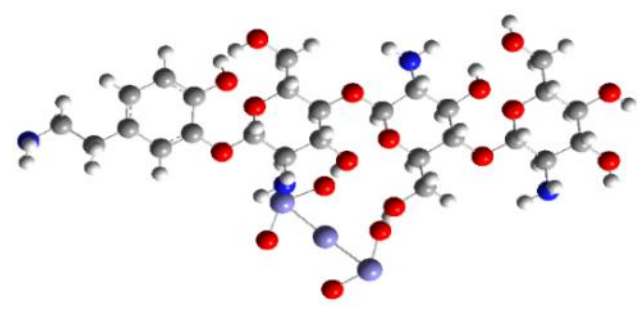

b-

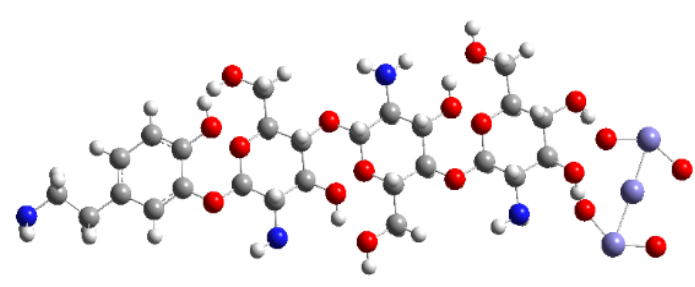

d-

Figure 2. Molecular model molecules of chitosan-dopamine-iron at four proposed positions dopamine calculated at B3LYP/6-31G (d,p).

Geometry optimization calculations for the built-up model molecules of chitosan, dopamine, a blend of chitosan-dopamine, and chitosan-dopamine-iron were conducted at PM6 level. To promote the preferred position of $\mathrm{Fe}_{3} \mathrm{O}_{4} \mathrm{NPs}$, the total dipole moment and HOMO/LUMO band gap energy were calculated.

\subsection{Total dipole moment and HOMO/LUMO bandgap energy.}

Geometry optimization calculations are carried out to investigate the impact of the addition of $\mathrm{Fe} 3 \mathrm{O} 4$ NPs on the chitosan-dopamine blend's physical and biomedical features. Such computations are conducted via the DFT level at B3LYP/6-31G (d,p) method. Table 1 presents the total dipole moment and HOMO/LUMO band gap energy $(\Delta \mathrm{E})$ of the suggested structures.

Table 1. PM6 computed QSAR parameters, including charge total dipole moment (TDM) as Debye and HOMO/LUMO bandgap energy.

\begin{tabular}{l|c|c} 
Structure & TDM (Debye) & $\boldsymbol{\Delta E}(\mathbf{e V})$ \\
\hline Chitosan & 2.385 & 9.977 \\
\hline Dopamine & 1.837 & 8.629 \\
\hline Chitosan /dopamine & 4.444 & 8.911 \\
\hline Chitosan / dopamine- $\left(\mathrm{Fe}_{3} \mathrm{O}_{4}\right) 1$ & 4.408 & 8.372 \\
\hline Chitosan / dopamine- $\left(\mathrm{Fe}_{3} \mathrm{O}_{4}\right) 2$ & 6.283 & 8.744 \\
\hline Chitosan / dopamine- $\left(\mathrm{Fe}_{3} \mathrm{O}_{4}\right) 3$ & 10.800 & 7.034 \\
\hline Chitosan / dopamine- $\left(\mathrm{Fe}_{3} \mathrm{O}_{4}\right) 4$ & 4.783 & 8.947
\end{tabular}

It is well known that the HOMO/LUMO band gap represents the amount of energy required for exciting some electrons from the highest occupied molecular orbital (HOMO) to the lowest unoccupied molecular orbital (LUMO). Hence, it reflects the ease of having a conductive material. Therefore, bandgap energy can reflect the electrical activity of a substance. The chitosan-dopamine-iron blend's lowest resultant bandgap is at a third suggested position, which equals $7.034 \mathrm{eV}$. This reflects an increment in its electrical conductivity upon 
the addition of $\mathrm{Fe}_{3} \mathrm{O}_{4}$ NPs at this position. Hence, the addition of $\mathrm{Fe}_{3} \mathrm{O}_{4}$ NPs to chitosandopamine at the other suggested position has no noticeable effect on the blend conductivity.

Figure 3 illustrates HOMO and LUMO orbitals maps for chitosan-dopamine-iron for the proposed places of the iron addition. HOMO orbitals are homogenously concentrated in the vicinity of the pyrimidine part of $\mathrm{Fe}_{3} \mathrm{O}_{4}$ NPs structure. Similarly, the LUMO orbitals are shown to be distributed asymmetrically around the terminal of $\mathrm{Fe}_{3} \mathrm{O}_{4} \mathrm{NPs}$. Hence, $\mathrm{Fe}_{3} \mathrm{O}_{4} \mathrm{NPs}$ increases the blend's bioactivity, which mainly depends on HOMO/LUMO bandgap energy.

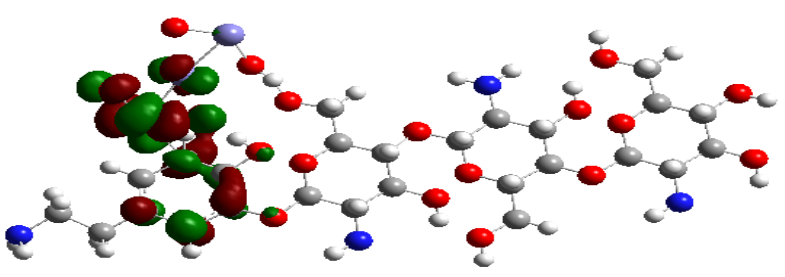

HOMO

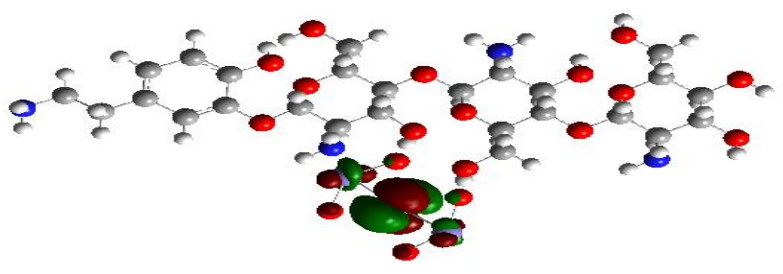

HOMO

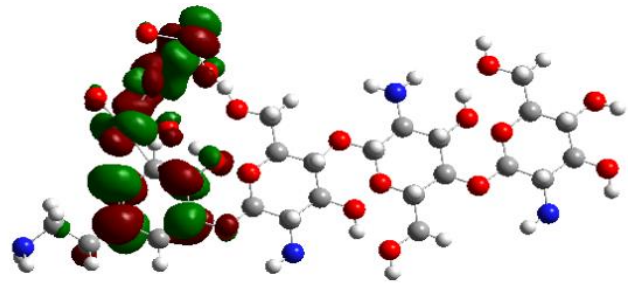

LUMO

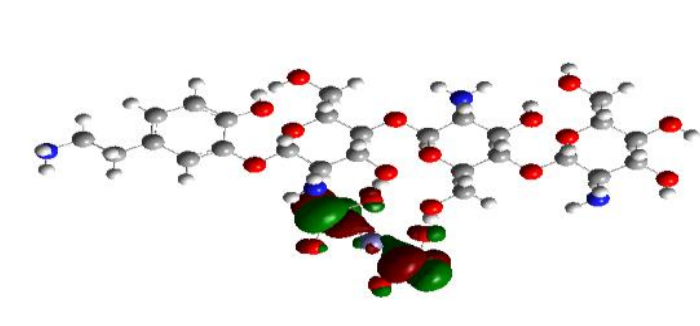

LUMO

a-

b-

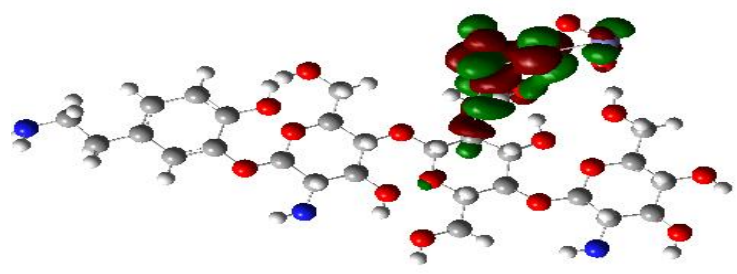

HOMO

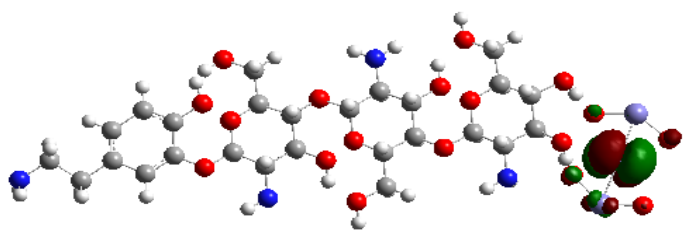

HOMO

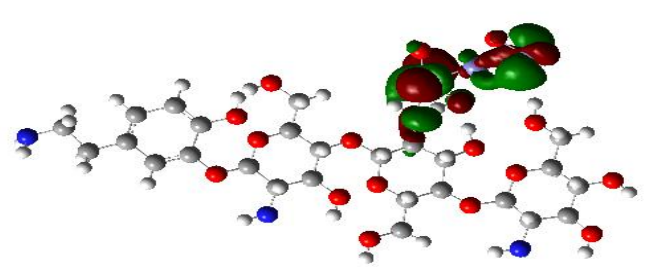

LUMO

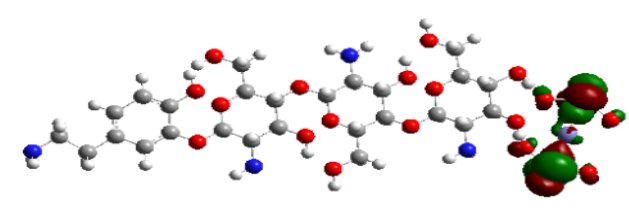

LUMO

d-

Figure 3. The mapped HOMO and LUMO orbitals for chitosan-dopamine-iron at the proposed places of the iron addition.

Table 1 presents a comparison between the B3LYP/6-31G(d,p) calculated the total dipole moment for chitosan, dopamine, chitosan-dopamine, chitosan- dopamine-iron 1, chitosan-dopamine-iron 2, chitosan-dopamine- iron 3, and chitosan-dopamine-iron 4 . The total dipole moment is always considered an excellent reference for the reactivity of chemical structures. It is clear that the addition of $\mathrm{Fe}_{3} \mathrm{O}_{4} \mathrm{NPs}$ at the third position increases the calculated 
total dipole moment, as indicated in table 1, while the bandgap energy is the lowest one. Increasing the total dipole moment indicates increasing the chitosan-dopamine-iron reactivity due to a $\mathrm{Fe}_{3} \mathrm{O}_{4} \mathrm{NPs}$ addition at this position.

\subsection{QSAR descriptors of chitosan, dopamine, and iron oxide nanoparticles.}

Quantitative structure-activity relationship (QSAR) presents a simple and accessible scheme for forecasting the biological activities of structures. It lasts to be one of the hottest topics of research work when judging biological activity [31,32]. QSAR descriptors are calculated for the proposed structures at the PM6 theoretical level. Table 2 presents some of the considered physical parameters, such as total energy E. Also, it presents the computed QSAR descriptors such as final heat of formation (FF), ionization potential (IP), Log P, molecular weight (MW), surface area (SA), and volume (V). Considered structures are all in the ground state; therefore, they all possess no electric charge and are neutral.

Table 2. Some of the calculated QSAR properties for chitosan-dopamine with the interaction of $\mathrm{Fe}_{3} \mathrm{O}_{4} \mathrm{NP}_{\text {for }}$ different positions at PM6.

\begin{tabular}{l|l|l|l|l|l|l|l} 
Structure & $\mathbf{C}$ & $\mathbf{E}(\mathbf{k c a l} / \mathbf{m o l})$ & $\mathbf{F F}(\mathbf{k c a l} / \mathbf{m o l})$ & $\mathbf{I P}(\mathbf{e V})$ & $\log \mathbf{P}$ & $\mathbf{M R}$ & $\mathbf{M W}(\mathbf{a u})$ \\
\hline Chitosan & 0.000 & -195539.421 & -578.295 & -9.720 & -5.236 & 105.723 & 501.483 \\
\hline Dopamine & 0.000 & -53715.793 & -67.794 & -8.657 & 0.847 & 42.674 & 153.178 \\
\hline Chitosan/dopamine & 0.000 & -240740.309 & -589.683 & -8.964 & -4.092 & 144.905 & 636.646 \\
\hline Chitosan/ dopamine- $\left(\mathbf{F e}_{3} \mathbf{O}_{4}\right) \mathbf{1}$ & 0.000 & -313634.074 & -906.119 & -8.755 & -3.008 & 150.305 & 868.179 \\
\hline Chitosan/dopamine- $\left(\mathbf{F e}_{3} \mathbf{O}_{4}\right) \mathbf{2}$ & 0.000 & -317170.732 & -873.230 & -9.106 & -3.008 & 150.305 & 868.179 \\
\hline Chitosan/dopamine- $\left.\mathbf{F e}_{3} \mathbf{O}_{4}\right) \mathbf{3}$ & 0.000 & -314912.915 & -827.608 & -7.258 & -2.586 & 147.011 & 868.179 \\
\hline Chitosan/dopamine $\left(\mathbf{F e}_{3} \mathbf{O}_{4}\right) \mathbf{4}$ & 0.000 & -312151.425 & -871.204 & -8.964 & -3.008 & 150.305 & 868.179
\end{tabular}

Total energy (E) is calculated as a good indicator of the stability of calculated compounds. Interaction of chitosan and dopamine with iron oxide nanoparticles significantly impacted energy. Its value was remarkably reduced after a $\mathrm{Fe}_{3} \mathrm{O}_{4}$ NPs addition at all the suggested positions reflecting a highly stable structure. There is a little difference in energy values between the four proposed $\mathrm{Fe}_{3} \mathrm{O}_{4}$ NPs sites. The calculated FF of chitosan-dopamineiron at the four proposed positions is lower than that of chitosan-dopamine, where their values are around $-873.230 \mathrm{kcal} / \mathrm{mol}$ and for chitosan-dopamine is $-589.683 \mathrm{kcal} / \mathrm{mol}$. Ionization potential is one of the QSAR descriptors that reflects chemical structures' electrical conductivity and hence their reactivity. The resultant IP of Chitosan-dopamine and Chitosandopamine-iron indicates that the interaction of Chitosan-dopamine with iron oxide nanoparticles has no significant impact on IP values. However, the addition of $\mathrm{Fe}_{3} \mathrm{O}_{4} \mathrm{NPs}$ at the third position increases the calculated IP as indicated in table 2, reflecting its high reactivity. $\log \mathrm{P}$ is the abbreviation for the partition coefficient's logarithm, which is useful in detecting whether the compound is hydrophilic or hydrophobic. The positive values indicate hydrophobic structures, and the negative ones reflect hydrophilic structures. Results in table 2 ensure that chitosan-dopamine and chitosan-dopamine-iron are hydrophilic structures. Therefore, the degradation process of chitosan-dopamine and chitosan-dopamine-iron involves the use of water to breakdown the polymer backbone until monomers are obtained.

Molecular weight (MW) is also calculated for chitosan-dopamine and chitosandopamine-iron. MW of chitosan-dopamine is lower than that of chitosan-dopamine-iron as a reasonable result for the addition of iron oxide nanoparticles. Surface area and volume are calculated as one of the geometrical QSAR descriptors that are usually conducted to evaluate the effect of chitosan-dopamine interaction with iron oxide nanoparticles. The surface area and volume of Chitosan-dopamine-iron are greater than of chitosan-dopamine. Polarizability (P) 
can be defined as the ease of a chemical structure to be polarized in response to external forces. It somehow reflects the reactivity of chemical structures, and it depends on the volume of them. The interaction of chitosan-dopamine with iron oxide nanoparticles has a significant effect on the resultant structures' polarizability. The polarizability of chitosan-dopamine-iron is greater than that of chitosan-dopamine.

3.4. Molecular electrostatic potential (MESP) maps of chitosan, dopamine, and iron oxide nanoparticles.

Calculations were carried out for chitosan, dopamine, a blend of chitosan-dopamine, and chitosan-dopamine-iron structures utilizing high theoretical DFT levels B3LYP/6-31G $(\mathrm{d}, \mathrm{p})$ method. Such computations were processed in order to map their molecular electrostatic surface potential (MESP). The resultant MESP maps are recognized by comprising of various colors ranging from red to dark blue, indicating the extreme negative and positive sites, respectively, in the interesting structure. The completed map configuration includes colors in the order of red, yellow, green, light blue, and dark blue from the most negative to most positive. Red color refers to extreme negative potentials while dark blue for extreme positive potentials. The yellow color is a less negative potential area with respect to the red one.

Similarly, light blue regions are of less positive potentials than dark blue. Green color refers to regions of nearly neutral potentials relative to both red and dark blue. The distribution of potentials and colors can be linked in some way by the relevance between the electronegativity of the bonded atoms. Highly electronegative atoms will be colored by red when connected with less electronegative ones having atoms of close electronegative values create the color distribution to be much tight. MESP maps of all suggested structures were constructed.

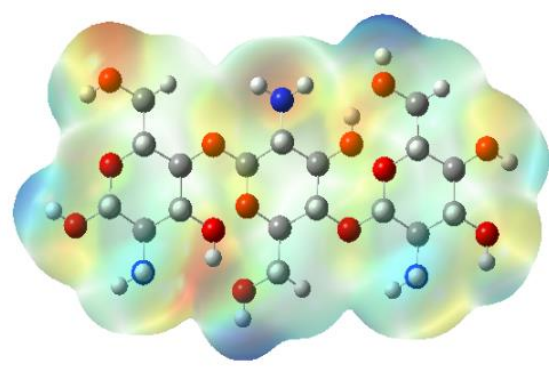

a-

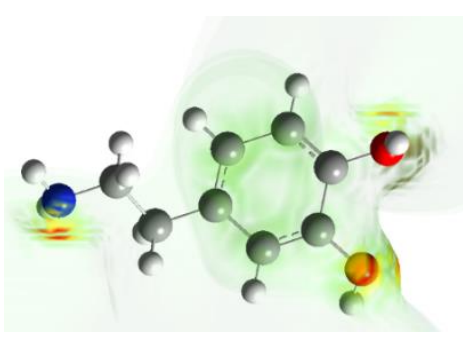

b-

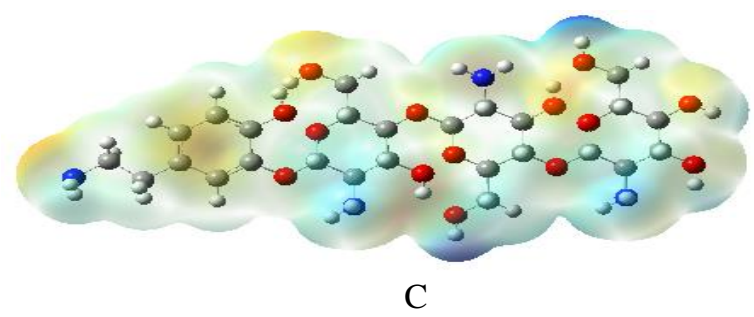

Figure 4. Calculated MESP maps of chitosan (a), dopamine (b), and a blend of chitosan and dopamine(c) at DFT level using B3LYP/6-31G (d,p) method. Different colors indicate different electronegative regions.

Figure 4 demonstrates the resultant MESP maps as 3D surface of chitosan (a), dopamine (b), and a blend of chitosan-dopamine (c). Figure 4 (a) consists of three main colors; yellow, light blue, and red colors; yellow at the two terminals around the two $\mathrm{O}$ atoms. The 
light blue color, which is concentrated at the center of the benzene rings, and red color around the $\mathrm{O}$ atom between the first and second unit of chitosan.

Although the $\mathrm{O}$ atom is highly electronegative, it appears in yellow at the terminal instead of the usual red color. This may be attributed to two $\mathrm{O}$ atoms' presence in two opposite directions canceling their electronegativity effect. Figure 4 (b) contains only two colors; the green color surrounding the benzene ring at the center and the yellow one in the terminal two O atoms' vicinity. Similarly, figure 4 (c) has two colors. Light blue is dominant on the structure, and a yellow color appears at different positions of this figure. This suggests the structure most probable to undergo electrophilic interactions. Figure 5 demonstrates the resultant MESP maps as a contour for the previously mentioned structures; chitosan (a), dopamine (b), and a blend of chitosan-dopamine (c). This figure confirms the same behavior for the various atoms.

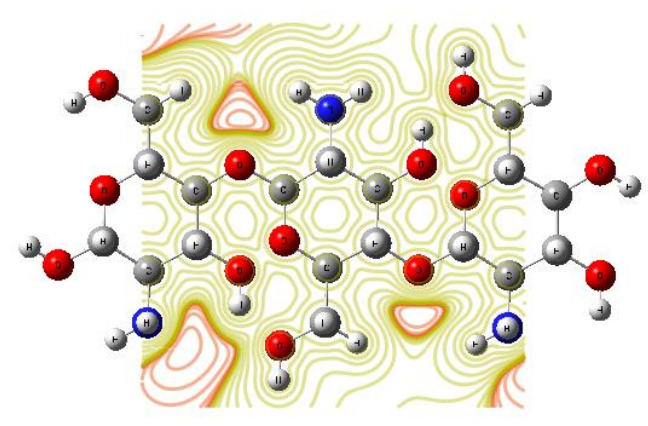

a-

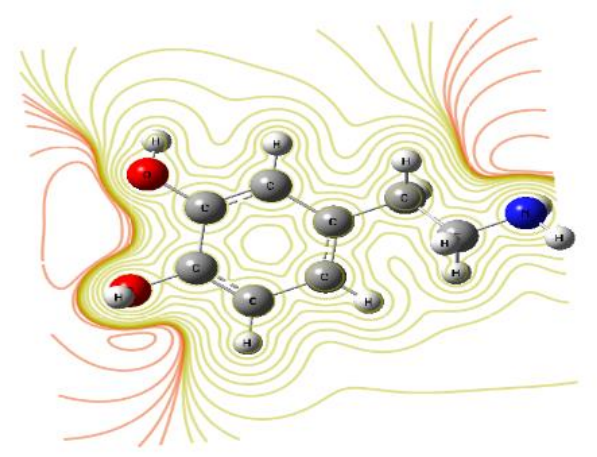

b-

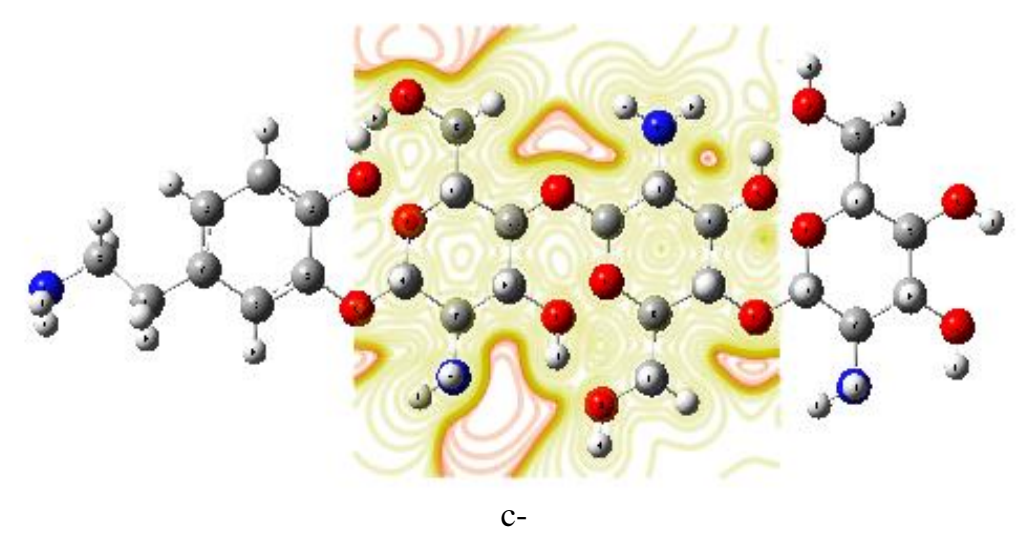

Figure 5. Electrostatic potential maps of chitosan (a), dopamine (b), and a blend of chitosan - dopamine(c) as a contour.

Figure 6 demonstrates the resultant MESP maps of chitosan-dopamine-iron structures at four suggested positions of $\mathrm{Fe}_{3} \mathrm{O}_{4}$ NPs (as 3D surface). We calculated MESP for chitosan and dopamine with $\mathrm{Fe}_{3} \mathrm{O}_{4}$ NPs on the same previous level of theory. The MESP maps consist of four main colors; red, yellow, light, and dark blue. The red color appears at the center of a benzene ring of dopamine. Light blue color manifests at almost all sites of the structure, and yellow color appears as a cloud around the dopamine structure, and dark blue display at $\mathrm{Fe}$ atoms. MESP contour maps are illustrated in figure 7. Red electronegative lines surround $\mathrm{O}$ atoms and yellow lines surrounding other atoms. 


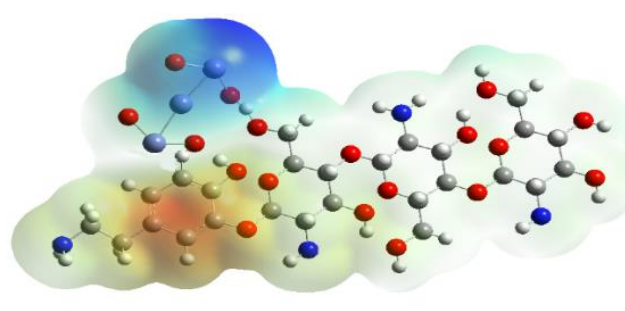

a-

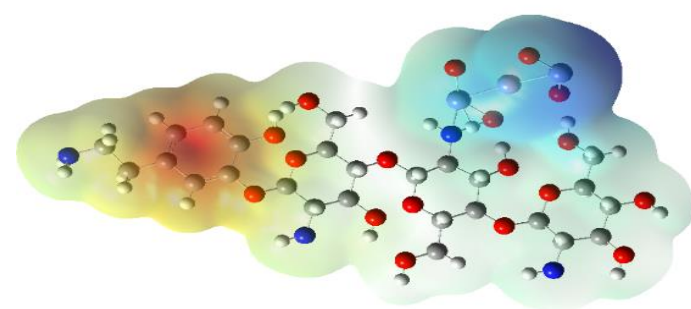

c-

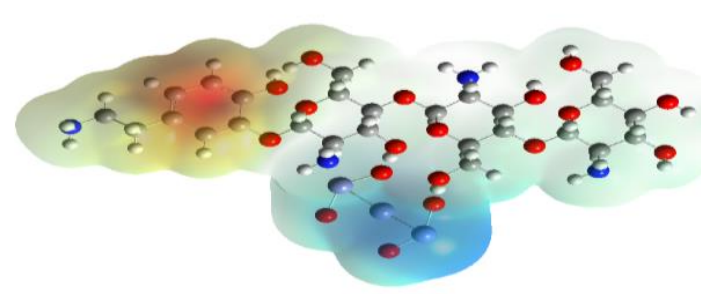

b-

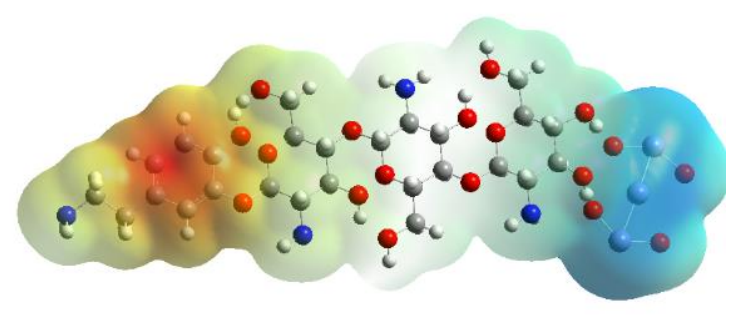

d-

Figure 6. Calculated MESP maps as a 3D surface for chitosan-dopamine-iron at four proposed positions at DFT level using B3LYP/6-31G $(\mathrm{d}, \mathrm{p})$ method.

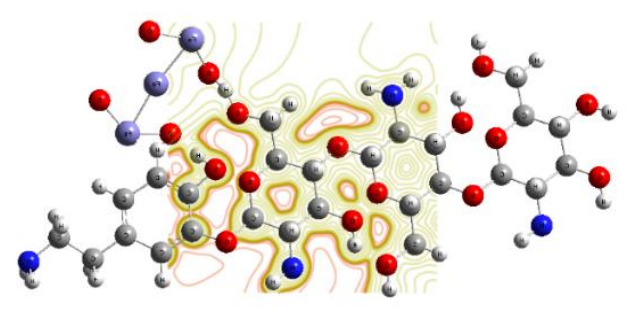

a-

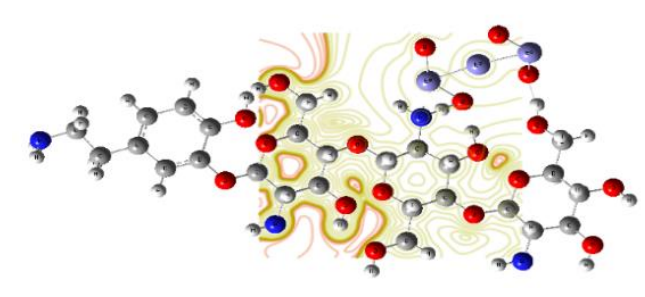

c-

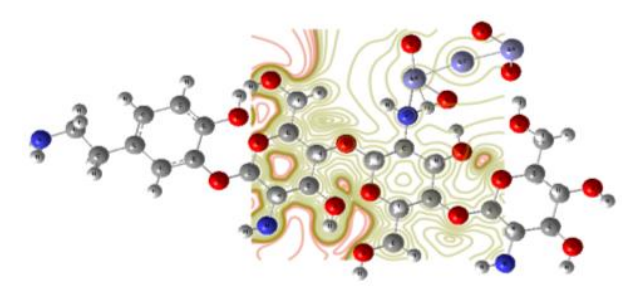

b-

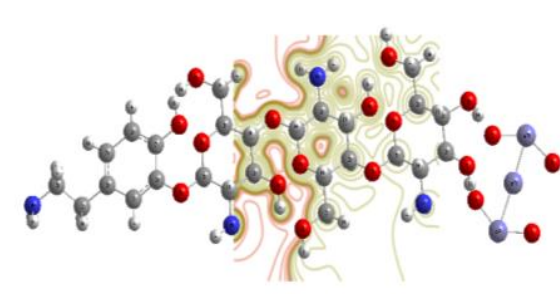

d-

Figure 7. Electrostatic potential maps as a contour for chitosan-dopamine-iron at four proposed positions.

\section{Conclusions}

This research work investigated the QSAR parameters and constructed molecular electrostatic potential (MESP) maps of chitosan, dopamine. A blend consists of them and the suggested blend with $\mathrm{Fe}_{3} \mathrm{O}_{4}$ NPs at various positions. QSAR parameters are useful in quantifying the biological activities of a chitosan-dopamine-iron blend. $\log \mathrm{P}$ of the chitosandopamine-iron blend interaction produces a slightly hydrophilic chemical structure, which means this blend will degrade by hydrolytic degradation. 
MESP maps were executed at a high theoretical DFT level using the B3LYP/6-31G (d,p) method. The linkage between TDM and the constructed MESPs was also analyzed. Four different sites of $\mathrm{Fe}_{3} \mathrm{O}_{4}$ NPs with the blend were built up. MESP maps can explore the nature of chemical addiction, through which a molecule is most probable to undergo; either electrophilic or nucleophilic addition. Results show that the suggested blend most likely to undergo electrophilic interactions. The obtained PM6 model indicated that blending chitosan with dopamine increased the blend's overall reactivity by increasing the total dipole moment of the blend to a greater value than that of individual polymers. In addition, the third suggested site of $\mathrm{Fe}_{3} \mathrm{O}_{4} \mathrm{NPs}$ has the largest value of TDM, which means that it has the highest stability and largest reactivity.

\section{Funding}

This research received no external funding.

\section{Acknowledgments}

The authors have to obvious their assessment to professor A. A. Hamza Professor of Physics, Ex-president of British University in Egypt, and Mansoura University for his worthy discussion during this study.

\section{Conflicts of Interest}

The authors declare no conflict of interest.

\section{References}

1. Ghosh, S.; Cabral, J.D.; Hanton, L.R.; Moratti, S.C. Strong poly (ethylene oxide) based gel adhesives via oxime cross-linking. Acta Biomater. 2016, 29, 206-14, https://doi.org/10.1016/j.actbio.2015.10.018.

2. Park, K.H.; Seong, K.Y., Yang, S.Y.; Seo, S. Advances in medical adhesives inspired by aquatic organisms' adhesion. Biomater. Res. 2017, 21, 1-9, https://doi.org/10.1186/s40824-017-0101-y.

3. El-Bakary, M.; El-Farahaty, K.; El-Sayed, N. In vitro degradation characteristics of polyglycolic/polycaprolactone (PGA/PCL) copolymer material using Mach-Zehnder interferometer. Mater. Res. Express. 2019, 6, https://doi.org/10.1088/2053-1591/ab4307.

4. El-Farahaty, K.A.; El-Bakary, M.A.; El-Sayed, N.M. The effect of different thermal treatment on the physical properties of PGA/PCL copolymer suture material. Microscopy Research and Technique 2018, 81, 64-73, https://doi.org/10.1002/jemt.22957.

5. Ates, B.; Koytepe, S.; Balcioglu, S.; Karaaslan, M.G.; Kelestemur, U.; Gulgen, S.; Ozhan, O. Biomimetic approach to tunable adhesion of polyurethane adhesives through $\mathrm{Fe} 3+$ cross-linking and hydrophobic tween units with balance of adhesion/cohesion forces. International Journal of Adhesion and Adhesives 2019, 95, https://doi.org/10.1016/j.ijadhadh.2019.102396.

6. Bhagat, V.; Becker, M.L. Degradable Adhesives for Surgery and Tissue Engineering. Biomacromolecules 2017, 18, 3009-3039, https://doi.org/10.1021/acs.biomac.7b00969.

7. Lu, M.M.; Bai, J.; Shao, D.; Qiu, J.; Li, M.; Zheng, X.; Xiao, Y.; Wang, Z.; Chang, Z.M.; Chen, L.; Dong, W.F.; Tang, C.B. Antibacterial and biodegradable tissue nano-adhesives for rapid wound closure. Int $J$ Nanomedicine 2018, 13, 5849-5863, https://doi.org/10.2147/IJN.S177109.

8. Du, X.; Wu, L.; Yan, H.; Qu, L.; Wang, L.; Wang, X.; Ren, S.; Kong, D.; Wang, L. Multifunctional Hydrogel Patch with Toughness, Tissue Adhesiveness, and Antibacterial Activity for Sutureless Wound Closure. ACS Biomaterials Science \& Engineering 2019, 5, 2610-2620, https://doi.org/10.1021/acsbiomaterials.9b00130.

9. Ryu, J.H.; Lee, Y.; Kong, W.H.; Kim, T.G.; Park, T.G.; Lee, H. Catechol-Functionalized Chitosan/Pluronic Hydrogels for Tissue Adhesives and Hemostatic Materials. Biomacromolecules 2011, 12, 2653-2659, https://doi.org/10.1021/bm200464x.

10. Li, Q.; Barrett, D.G.; Messersmith, P.B.; Holten-Andersen, N. Controlling Hydrogel Mechanics via BioInspired Polymer-Nanoparticle Bond Dynamics. ACS Nano 2016, 10, 1317-1324, https://doi.org/10.1021/acsnano.5b06692. 
11. Pramudya, I.; Rico, C.G.; Lee, C.; Chung, H. POSS-Containing Bioinspired Adhesives with Enhanced Mechanical and Optical Properties for Biomedical Applications. Biomacromolecules 2016, 17, 3853-3861, https://doi.org/10.1021/acs.biomac.6b00805.

12. Lu, D.; Zhang, Y.; Li, T.e.; Li, Y.; Wang, H.; Shen, Z.; Wei, Q.; Lei, Z. The synthesis and tissue adhesiveness of temperature-sensitive hyperbranched poly(amino acid)s with functional side groups. Polymer Chemistry 2016, 7, 1963-1970, https://doi.org/10.1039/C5PY01844G.

13. Lih, E.; Lee, J.S.; Park, K.M.; Park, K.D. Rapidly curable chitosan-PEG hydrogels as tissue adhesives for hemostasis and wound healing. Acta Biomaterialia 2012, 8, 3261-3269, https://doi.org/10.1016/j.actbio.2012.05.001

14. Matsuda, M.; Inoue, M.; Taguchi, T. Adhesive properties and biocompatibility of tissue adhesives composed of various hydrophobically modified gelatins and disuccinimidyl tartrate. Journal of Bioactive and Compatible Polymers 2012, 27, 481-498, https://doi.org/10.1177\%2F0883911512455116.

15. Frost, S.J.; Mawad, D.; Higgins, M.J.; Ruprai, H.; Kuchel, R.; Tilley, R.D.; Myers, S.; Hook, J.M.; Lauto, A. Gecko-inspired chitosan adhesive for tissue repair. NPG Asia Materials 2016, 8, https://doi.org/10.1038/am.2016.73.

16. Ibrahim, M.; Saleh, N.; Elshemey, W.; Elsayed, A. Hexapeptide functionality of cellulose as NS3 protease inhibitors. Med. Chem. 2012, 8, 826-30, https://doi.org/10.2174/157340612802084144.

17. Assirey, E.A.; Sirry, S.M.; Burkani, H.A.; Ibrahim, M.A. Modified Ziziphus spina-christi stones as green route for the removal of heavy metals. Scientific Reports 2020, 10, https://doi.org/10.1038/s41598-02076810-y.

18. Saji, R.S.; Prasana, J.C.; Muthu, S.; George, J.; Kuruvilla, T.K.; Raajaraman, B.R. Spectroscopic and quantum computational study on naproxen sodium. Spectrochimica Acta Part A: Molecular and Biomolecular Spectroscopy 2020, 226, https://doi.org/10.1016/j.saa.2019.117614.

19. Dinparast, L.; Hemmati, S.; Alizadeh, A.A.; Zengin, G.; Kafil, H.S.; Bahadori, M.B.; Dastmalchi, S. An efficient, catalyst-free, one-pot synthesis of $4 \mathrm{H}$-chromene derivatives and investigating their biological activities and mode of interactions using molecular docking studies. Journal of Molecular Structure 2020, 1203, https://doi.org/10.1016/j.molstruc.2019.127426.

20. Ezzat, H.; Menazea, A.A.; Omara, W.; Basyouni, O.H.; Helmy, S.A.; Mohamed, A.A.; Tawfik, W.; Ibrahim, M. DFT:B3LYP/ LANL2DZ Study for the Removal of Fe, Ni, Cu, As, Cd and Pb with Chitosan. Biointerface Res. Appl. Chem. 2020, 10, 7002-7010, https://doi.org/10.33263/BRIAC106.70027010

21. Refaat, A.; Ibrahim, M.A.; Elhaes, H.; Badry, R.; Ezzat, H.; Yahia, I.S.; Zahran, H.Y.; Shkir, M. Geometrical, vibrational, and physical properties of polyvinyl chloride nanocomposites: Molecular Modeling Approach. J. Theor. Comput. Chem. 2020, 18.

22. Saleh, N.A.; Elhaes, H.; Ibrahim, M. Design and development of some viral protease inhibitors by QSAR and molecular modeling studies. In: Viral proteases and their inhibitors. 1st Edition, Elsevier; 2017; pp. 2558, https://doi.org/10.1016/B978-0-12-809712-0.00002-2.

23. Bulat, F.A.; Toro-Labbé, A.; Brinck, T.; Murray, J.S.; Politzer, P. Quantitative analysis of molecular surfaces: areas, volumes, electrostatic potentials and average local ionization energies. Journal of Molecular Modeling 2010, 16, 1679-1691, https://doi.org/10.1007/s00894-010-0692-x.

24 Fahim, A.M.; Shalaby, M.A.; Ibrahim, M.A. Microwave-assisted synthesis of novel 5-aminouracil-based compound with DFT calculations. Journal of Molecular Structure 2019, 1194, 211-226, https://doi.org/10.1016/j.molstruc.2019.04.078

25. Mahmoud, A.A.; Osman, O.; Elhaes, H.; Ferretti, M.; Fakhry, A.; Ibrahim, M.A. Computational Analyses for the Interaction Between Aspartic Acid and Iron. Journal of Computational and Theoretical Nanoscience 2018, 15, 470-473, https://doi.org/10.1166/jctn.2018.7113.

26. El-Mansy, M.A.; Bayoumy, A.M.; Ezzat, H.; El-Sayed, N.; Elhaes, H.; Ibrahim, M.A. Modeling the Effect of Hydration on the Electronic and Vibrational Properties of AZT. Biointerface Res. Appl. Chem. 2021, 11, 9253-9265, https://doi.org/10.33263/briac112.92539265.

27. Galal, A.M.F.; Atta, D.; Abouelsayed, A.; Ibrahim, M.A.; Hanna, A.G. Configuration and molecular structure of 5-chloro-N-(4-sulfamoylbenzyl) salicylamide derivatives. Spectrochimica Acta Part A: Molecular and Biomolecular Spectroscopy 2019, 214, 476-486, https://doi.org/10.1016/j.saa.2019.02.070.

28. El-Barbary, A.; El-Nahass, M.; Kamel, M.; El-Mansy, M.A.M. FT-IR, FT-Raman spectra and ab initio HF, DFT vibrational analysis of P-methyl acetanilide. J. Raman Spectrosc. 2009, 11, 1977-1987.

29. El-Mansy, M.; El-Bana, M.; Fouad, S. On the spectroscopic analyses of 3-Hydroxy-1-Phenyl-Pyridazin-6 (2H) one (HPHP): A comparative experimental and computational study. Spectrochim. Acta Part A 2017, 176, 99-105, https://doi.org/10.1016/j.saa.2016.12.040.

30. Ibrahim, M.; El-Haes, H. Computational Spectroscopic Study of Copper, Cadmium, Lead and Zinc Interactions in the Environment. Int. J. Environ. Pollut. 2005, 23, 417-424, https://doi.org/10.1504/IJEP.2005.007604.

31. Ibrahim, M.; Mahmoud, A.A. Computational Notes on the Reactivity of some Functional Groups. J. Comput. Theor. Nanosci. 2009, 6, 1523-1526, https://doi.org/10.1166/jctn.2009.1205. 
32. Politzer, P.; Laurence, P.R.; Jayasuriya, K. Molecular electrostatic potentials: an effective tool for the elucidation of biochemical phenomena. Environ. Health Persp. 1985, 61, 191-202, https://doi.org/10.1289/ehp.8561191.

33. Şahin, Z.S.; Şenöz, H.I.; Tezcan, H.; Büyükgüngör, O. Synthesis, spectral analysis, structural elucidation and quantum chemical studies of (E)-methyl-4-[(2-phenylhydrazono)methyl]benzoate. Spectrochim. Acta A 2015, 143, 91-100, https://doi.org/10.1016/j.saa.2015.02.032.

34. Frisch, M.; Trucks, G.; Schlegel, H.B.; Scuseria, G.E.; Robb, M.A.; Cheeseman, J.R.; Scalmani, G.; Barone, V.; Mennucci, B.; Petersson, G.J.I. Gaussian, Inc., Wallingford CT, 2010.

35. Becke, A.D. Density-functional thermochemistry. III. The role of exact exchange. The Journal of Chemical Physics 1993, 98, 5648-5652, https://doi.org/10.1063/1.464913.

36. Lee, C.; Yang, W.; Parr, R.G. Development of the Colle-Salvetti correlation-energy formula into a functional of the electron density. Physical Review B 1988, 37, 785-789, https://doi.org/10.1103/PhysRevB.37.785.

37. Miehlich, B.; Savin, A.; Stoll, H.; Preuss, H. Results obtained with the correlation energy density functionals of becke and Lee, Yang and Parr. Chemical Physics Letters 1989, 157, 200-206, https://doi.org/10.1016/0009-2614(89)87234-3. 